\author{
Mingjun HE, Jianjun MU*, Fuqiang \\ LIU, Keyu REN, Yang WANG and \\ Tongshuai GUO \\ Department of Cardiology, The First Affiliated \\ Hospital, Medical School of Xi'an Jiaotong \\ University, Xi'an 710061, China
}

Dates: Received: May 06, 2014; Accepted: June 16, 2014; Published: June 18, 2014

*Corresponding author: Jianjun MU, The

First Affiliated Hospital, Medical School of Xi'an Jiaotong University, Xi'an 710061, China, Tel: +86-029-85323800; Email: Mujjun@163.com

www.peertechz.com

Keywords: Resistant hypertension; Diagnosis; Treatment

ISSN: 2455-2976

\author{
Short Communication
}

\section{Progression in Diagnosis and Treatment of Resistant Hypertension}

\section{Abbreviations}

RH: Resistant Hypertension; BP: Blood Pressure; SBP: Systolic Blood Pressure; DBP: Diastolic Blood Pressure; DM: Diabetes Mellitus; OSA: Obstructive Sleep Apnea; PA: Primary Aldosteronism; RDN: Renal Denervation; CPAP: Continuous Positive Airway Pressure

\section{Introduction}

Resistant Hypertension (RH) is a major risk factor of numerous diseases, resulting in increased incidence of ischemic heart disease, heart failure, cerebrovascular events, and renal dysfunction. Hypertension is defined as resistant to treatment when a therapeutic strategy that includes appropriate lifestyle measures plus a diuretic and two other antihypertensive drugs belonging to different classes at adequate doses (but not necessarily including a mineralocorticoid receptor antagonist) fails to lower $\mathrm{BP}$ values to $<140 / 90 \mathrm{mmHg}$, then the hypertension can be defined as RH [1].

Although with affluent knowledge on the management of hypertension and several effective antihypertensive drugs, RH is still a challenging clinical problem. Many factors including measurement error, poor drug adherence, inappropriate drug intakes, secondary hypertension, white-coat hypertension and masked hypertension seriously affect blood pressure (BP) control. Correct diagnosis and appropriate drug therapy are pretty important to get BP controlled. Interventional therapyprovides $\mathrm{RH}$ with another option. With the increase of population aging, incidence of diabetes mellitus (DM), obstructive sleep apnea (OSA) and renal diseases, the prevalence of RHrise constantly. This article aim to discuss the prevalence, diagnosis and treatment of RH to attract physicians' attention to adopt correct measures to diagnose and treat $\mathrm{RH}$.

\section{Discussion}

\section{Prevalence}

Strictly speaking, $\mathrm{RH}$ is not a definition of disease, but of management. So it is difficult to get its correct prevalence. Because of this, its prevalence is still unclear. Some large-scale clinical trials intended to figure out the prevalence of $\mathrm{RH}$ in overall population and hypertensive populationare shown below.

According to the latest data from ESH/ESC [1], the prevalence of $\mathrm{RH}$ is around $5 \%-30 \%$ of the overall hypertensive populationwithfigures less than $10 \%$ probably representing the true prevalence. Arepresentative survey carried out by American National Health and Nutrition Examination [2] including > 15000 non-pregnant adults between 2003 and 2008 found that resistant hypertension criteria were met in $8.9 \%$ of all individuals with hypertension, and in $12.8 \%$ of the hypertensive drugs treated population. A complete database for 7.9 years from Olivetti Heart Study (OHS) in southern Italy [3] showed that the prevalence of $\mathrm{RH}$ was $4.8 \%$ in the whole study population, and $10.1 \%$ in hypertensive participants. It seems that the prevalence ofRHis around $10 \%$ in hypertensive population, butsituations including treatment adherence, white coat hypertension and secondary hypertension were not reported. Another more detailed research by Stacie et al [4] demonstrated thatafter the exclusion of those who lost baseline data or failed to adhere to the research, the inadequate number of antihypertensive drugs, and poor medication adherence,the prevalence of true RH was only $1.9 \%$ among 205750 patients. It is, 
therefore, reasonable to believe that the prevalence of $\mathrm{RH}$ was much lower than we expected.

\section{Diagnosis}

American Heart Associationregards the diagnosis of $\mathrm{RH}$ as aprocedure of exclusion [5]. Hence,individuals are supposed to be evaluated carefully to exclude Pseudo and secondary hypertension.

\section{Common Pseudo-Resistance}

1) Measurement Error: Incorrect measurement leads to improper diagnosis and treatment. Attention should be paid to choose proper techniques for BP measuring. Common errors include inappropriate cuff size, incorrect patient's arm position, and inadequate rest before measurement. Moreover, arterial stiffness will make it easier to get a higher SBP in older people.

2) Poor drug therapy adherence: Patients' demographics, side effects, dosages, the cost and number of medications, as well as patients' knowledge, beliefs and attitudes about hypertension seriously result in poor drug therapy adherence. A latest clinical study has illustrated that $\mathrm{RH}$ patients just took less than $50 \%$ of the recommended maximal daily doses of their anti-hypertensive medications [6]. Besides, physicians' failure to comply with guidelines to pursue adequate dosage or category of anti-hypertensive drugs is alsoa significant reason leading touncontrolled hypertension. Because of the potential adverse effects of multiple drug combination or physicians' skepticism about the benefit of intensifying therapy, patients already on 3 or more antihypertensive medications are at an increased risk of poor treatment adherence and clinical inertia.

3) White Coat Hypertension and Masked Hypertension: Most patients have higher BP levels when measured by physicians than assessed by themselves or Ambulatory Blood Pressure Monitoring(ABPM). According to a study by Barochiner et al. [7], among 302 treated hypertensive patients, the prevalence of officeresistance hypertension was $27.6 \%$. Therefore, when BP assessed by oneself $135 / 85 \mathrm{mmHg}$, or by ABMP $130 / 80 \mathrm{mmHg}$ (in $24 \mathrm{~h}$ ), or $135 / 85 \mathrm{mmHg}$ (daytime), or $130 / 80 \mathrm{mmHg}$ (nighttime) plus office BP $140 / 90 \mathrm{mmHg}$, the diagnosis of hypertension is tenable.

There still exist an opposite phenomenon-masked hypertension (MH). MH is defined as a clinical condition in which a patient's office $\mathrm{BP}$ level is $<140 / 90 \mathrm{mmHg}$ but ambulatory or home BP readings are in the hypertensive range. The prevalence in the population is about the same as that of isolated office hypertension; about 1 in 7 or 8 persons with a normal office BP level may fall into this category. The high prevalence of $\mathrm{MH}$ would suggest the necessity for measuring out-of-office BP in persons with apparently normal or well-controlled office BP. Reactivity to daily life stressors and behavioral factors such as smoking, alcohol use, contraceptive use in women, and sedentary habits can selectively influence $\mathrm{MH}$. MH will influence the diagnosis and treatment of hypertension, and individuals have been shown to have greater-than-normal prevalence of organ damage, particularly with an increased prevalence of metabolic risk factors, left ventricular mass index, carotid intima-media thickness, and impaired large artery distensibility compared with patients with a truly normal BP level in and out of the clinic or office. Therefore, $\mathrm{MH}$ should be careful screened.

\section{The Exclusion of Secondary Hypertension}

Secondary hypertension includes some common diseases, such as obstructive sleep apnea (OSA), primary aldosteronism (PA), reno-vascular hypertension, and renal diseases etc. and some less common diseases e.g. aortic coarctation, cushing syndrome, pheochromocytoma, and thyroid disorders etc. $(<1 \%[8])$. Theymake up a high percentage of uncontrolled hypertension, and are prone to show the characteristic of "resistance".

1) Inappropriate drugintakes: $A$ large number of drugs or chemical substances can induce BP increase transiently or persistently, or significantly interfere with BP-lowering effects of antihypertensive drugs. Several pharmacological agents have a similar effect with RAAS to cause elevated BP and some substances even act directly on arteriolar structure to constrict blood vessels and raise BP. NSAID, corticosteroids, erythropoietin, sympathomimetic, antihistamines etc. are commonly inappropriate drugs according to Canto et al. [9]. Thus, interfering substances should be promptly discontinued or minimized.

2) Obstructive sleep apnea (OSA): Among the entire patients with uncontrolled secondary hypertension, OSA is the most common one [10]. Its association with hypertension is due to the sustained increaseof the sympathetic nervous system activity. Its prevalence is approximately $85 \%$ among the resistant-to-treatment patients [11]. The common characteristics of OSA are snoring that can be loud enough to disturb the bed partners' sleep and excessive daytime sleepiness. Cessation of breathing which ends by snorting sound canalso be found. Other symptoms are unexplained perversion of sleep, morning headache, memory problems and difficulties in concentrating. PSG (polysomnography), as the golden standard to diagnose OSA, should not be forgotten in patients with typical symptoms.

3) Primary aldosteronism (PA): PA caused by aldosteroneproducing adenoma or unilateral adrenal hyperplasia shows an increasing trend, with a reported prevalence of around $20 \%$ in resistant hypertensive patients [12], and the incidenceis higher in patients with Type 2 DM [13]. Maintained hypervolemia plays a key role in remaining resistant. Unexplained hypokalemia and hypertension are the typical symptoms of PA. Plasma renin activity (PRA), plasma aldosterone concentration (PAC) and a ratio of PRA to PAC (ARR) are sensitivity and specificity cluesto help to diagnose PA [14]. Other measures,for instance,24-hour urine electrolytes and adrenal computed tomography (CT)should be applied to identify PA.

4) Reno-vascular hypertension: Reno-vascular hypertension is defined as systemic hypertension resulting from renal arterial compromise, often due to occlusive lesions of the main renal arteries. Renal artery stenosis caused by fibro-muscular dysplasia (in young adults, especially women) or atherosclerotic renal artery stenosis (in 
elderly) will lead to activation of RAASand sodium retention, and then, at least in initial stage [15], result in Reno-vascular hypertension. The presence of an abdominal bruit should lead to a workup for Reno-vascular hypertension. A duplex Doppler flow study, magnetic resonance (MR) angiography with gadolinium enhancements and spiral CT scan, perhaps, are sensitive and specific screening tests.

5) Renal diseases: Chronic glomerular nephritis, diabetic nephropathy, chronic pyelonephritis and nephritis syndrome rank the firstfour common renal diseases. Volume expansion caused by impaired sodium handling, the activation of RAAS, alterations in endogenous vasodepressor compounds and possible promotion of activity of vasoactive substances lead to renal hypertension separately or interactively. It is supposed to be an apparent sign thatBP increase gradually within five or ten years after the original renal diseases. Besides a duplex Doppler flow study or CTisa necessity, especially for patients with renal dysfunction. Urinary VMA, serum, urinary catecholamine, adrenal CT, thyroid function, plasma cortisol and some other laboratory examinations should be taken into accountto exclude some rare secondary reasons.

\section{Treatment}

1. Life Intervention: In terms of the treatment of $\mathrm{RH}$,life intervention is the cornerstone. Measures, such as lowering body weightfor overweight patients $\left(\mathrm{BMI}<24 \mathrm{~kg} / \mathrm{m}^{2}\right)$,restricting alcohol intake foralcohol addictions (alcohol intake $<20-30 \mathrm{~g} / \mathrm{d}$ for male, $<10-15 \mathrm{~g} / \mathrm{d}$ for female) and abstaining from smoking should be adopted. Besides, easing off the life pressure and paying attention to psychological adjustment are also important.

Particularly, salt, the most important environmental factor of hypertension, plays a vital role in the occurrence and development of hypertension. Chronically high salt intake is very common among $\mathrm{RH}$ patients. However, it is still unclear how salt triggers and helps to maintain the increased BP. The possible mechanisms are high salt intake disrupt the balance of the central and autonomic nervous systems, hinder the ability of the kidney to mediate salt balance, cause the disorders of immune system andinterstitial hypertonicity in skin(help to increase body sodium retention) [16], and then help to maintain the high BP state. Besides, high salt intake can cause endothelial dysfunction, kidney function impairment (be independent of BP increase) which might participate in increasing the BP. Chronically high salt intake will seriously weaken the effect of lowering BP treatment, so salt restriction $(<6 \mathrm{~g} / \mathrm{d})$, potassium and magnesium supplement are recommended for everybody, especially for salt sensitive patients.

2. Pharmacological Therapy: Despite the availability of many effective antihypertensive drugs, drug therapy for $\mathrm{RH}$ remains a prominent problem. How to choose appropriate drugs is still a challenge for physicians. Besides, patients' burden affectsdrug adherence and then leads to weak effect and adherence.

1) Optimaldrug combination: The joint use of antihypertensive drugs of different mechanisms is the key pointof the treatment of RH. For high renin and high sympatheticactivity patients, RAS blockers
(ARB or ACEI) and $\beta$-blockers should be the first-line choice; for patients with hyperaldosteronism, spironolactone should be applied; for patients with excessive capacity and low circulating RAS, CCB and diuretics are the basic treatment. Sodium retention is one of the main reasons for $\mathrm{RH}$, so sufficient diuretics are often essential for $\mathrm{RH}$.

A retrospective study by Hanselin et al. [17] showed the use of antihypertensive drugs in $>140000$ patients with RH. They found that the most frequently used antihypertensive classes were RAS blockadesand diuretics, which account for $96.2 \%$ and $93.2 \%$ respectively, while CCB and $\beta$-blockers were $83.6 \%$ and $80.0 \%$, separately. Importantly, most patients were prescribed a combination ofRAS blockade (ACEI or ARB) plus CCB plus diuretic, which were considered an effective and generally well-tolerated drug combination. It is,therefore,recommended that the most reasonable drug combination solution for RH is RAS antagonist (ACEI or ARB) $+\mathrm{CCB}+$ diuretic, using the best tolerated dose.

On this basis, if BP still cannot be controlled at target levels, aldosterone antagonists (renal permitted) $25-50 \mathrm{mg} / \mathrm{d}$ or $\beta$-blockers, $\alpha-\beta$ receptor blockers or $\alpha$-blockers and central nervous system antagonist drugs can be added. Physicians should give priority to long-acting drugsbecause of the effect of recovering circadian rhythm, reducing morning surge, and providing 24-hour antihypertensive effect. Besides, compound preparation is widely used and recommended for their great tolerance and excellent antihypertensive effect.

2) Correct administration time: Taking medicine in correct time can notably lower morning surge and decrease BP variability. A work of 1306 subjects with true RH by Hermida et al. [18] illustrated that those ingesting at least one drug at morning time acquired significantly lower 24hour mean SBP/DBP(4.1/1.5 mmHg), which was more prominent in nighttime (SBP:9.7/DBP:4.4 mmHg). For this reason, appropriate administrationtime should not be ignored to allow patients to maximize profits.

3) Correct evaluation of drug adherence: Based on the appropriate drug combination, the evaluation of drug adherence is the prerequisite of effectiveness. The follow-up chat with patients and their family members every 2 to 4 weeks helps to gaindetails of the time, number, dosage, frequency, category, and habit of medicine intakes and to adjust drug prescriptions. Pulse measurement should be taken to reflect $\beta$-adrenergic receptor blocker intakes, and the test of blood uric acid and potassiumto reflect diuretic intakes. The drug compliance can be measured by blood drug concentration detection if necessary.

4) Efficacy and safety of drug prescriptions: Office BP measurements should be combined with home BP measurement or ABPM to evaluate the efficacy of a certain drug prescription. For patients with BP fluctuations, BP measurement before drug intakes,morning, midday and evening by patients themselves is necessary for following up. ABPMwhich can evaluate nocturnal $\mathrm{BP}$ isrecommended for those with huge BP fluctuations or great difference between office BPand home BP. During the whole 
therapeutic period, keeping in touch with your patients; being acquainted with any discomfort of them, especially positional vertigo and amaurosis. For those with renal dysfunction, or treated with high dose of RASI, aldosterone antagonists, loop-acting diuretics, periodic measurements of blood potassium, serum creatinine and creatinine clearance iscrucial.

3. Interventional Therapies (Device-Based Therapies): As the high risk of cardiovascular and cerebrovascular events resulting fromRH, BP control should be more strictly performed. In spite of life intervention and appropriate drug therapy, some high-risk patients still could not reach the standard. Regarding such patients, few options can be chosen to control the BP and lower the risk. Additional pharmacological strategies have been proposed. Devicebased therapies become more prevalent and have been studying recently.

1) Renal denervation (RDN)-a controversial method: Noradrenaline released by renal sympathetic nervesleads to the renal vasoconstriction, decreases the renal blood flow and GFR, and then subsequently enhances renin secretion, sodium and fluid reabsorption to elevate BP. A catheter-based radiofrequency ablation is a novel therapeutic strategy to selectively disrupt the renal sympathetic nerves.

(1) Early studies: Its efficacy and safety were supported by the Symplicity HTN1 and 2trials[19,20], which consist of RH patients who had a baseline systolic pressure $\geq 160 \mathrm{mmHg}(\geq 150 \mathrm{mmHg}$ in type $2 \mathrm{DM}$ patients). After six months, office BP values in the RDN group decreased by $32 / 12 \mathrm{mmHg}$, while no changes in control group showed. In the same time, ABPM over 24 hour, performing in a limited number $(n=20)$ of patients, showed a less pronounced reduction of $11 / 7 \mathrm{mmHg}$. The $\mathrm{BP}$ lowering effect couldextend to 24 months [20].

Limitations,such asthe small number of patients and short followup time, the lack ofcontrol group in Symplicity HTN-1 trial and sham control group in Symplicity HTN-2 trial, office-based measurement of BP were palpable in HTN 1 and 2 trails. Besides, white coat hypertension and secondary hypertension did not exclude strictly andlong-term BP reduction after RDNwas uncertain, which will have a great effect on evaluating the effect [19-21]. Although the above considerations, the European Society of Cardiology (ESC) [22] issued a consensus stated that RDN can be considered a therapeutic option in patients with $\mathrm{RH}$ who are not up to the required BP levelswith a combination of lifeintervention and pharmacologic therapy.

(2) Updated studies: For further evaluating the safety and effectiveness of RDN, the first blinded, randomized, controlled trial SYMPLICITY HTN-3 trail [23](including a sham-controlled group) was organized. Surprisingly, the trail met its primary safety endpoint (incidence of major adverse events), but it failed to meet its primary efficacy endpoint (change in office BP from baseline to six months).

All new technologies have ups and downs,soit should not be the end of RDN. Ample data have shownthat RDN reducedBP in animal models and in small human trials. In terms of this disappointing consequence, trusting on the outcome, further scrutinizing the data, choosing correct subgroup of RH patients (maybe only a part of hypertensive patients are suitable for this novel method for its various factors) should be the following steps. Besides, the equipments and methods of RDN (radiofrequency, high-frequency ultrasound, hypothermia, local injection of chemicals to ablate renal nerves) will also have a great impact on the effect, so it is possible that RDN with another device will be effective in treating hypertension.

2) Carotid Baroreflex Activation: Carotid sinuses play an important role in regulating autonomic nervous system, BP and intravascular volume. The carotid baroreflex is responsible for making adjustments to $\mathrm{BP}$ on a continuous minute-by-minute basis to prevent excessive rises and falls. The Rheos Baroreflex Hypertension Therapy System would help carotid sinuses itself to enhance the afferent nerve outflow from the baroreceptors to the cardiovascular control centers of the brain to produce the cancellated effect, which decreases heart rate (HR), myocardium contractility, vascular tone and increases the natriuresis to lower the arterial pressure[24,25]. The efficiency and safety were evaluated by a hypertension trial (DEBuT-HT) [26] conducted by a consortium of nine European institutions. This report provided clinical data from 2- and 3-year update, of the 45 patients enrolled in the study, 18 patients successfully completed this chronic therapy with the Rheos device for a mean duration of $58 \pm 6$ months. According to preliminary results of the Rheos Pivotal trial, the main adverse effects (incidence $\geq 2 \%$ ) includednerve damage (9\%); surgical (5\%), respiratory (2\%), and wound complications (2\%). Fortunately, most of the adverse effects would be fully recovered transiently [27]. However, just like RDN, its long-term efficiency and safety were still unknown. And because of its great expense, this treatment can only be a choice for RH.

3 Continuous Positive Airway Pressure (CPAP): OSA is a common reason for $\mathrm{RH}$. Their recurrent collapses of upper airway during sleep lead to hypoxic condition, a sustained activation of the sympathetic nervous system, an increased expression of systemic inflammatory markers, and derangement in endothelial function, which may result in increased BP, more cardiovascular and cerebrovascular events. CPAP, a new non-invasion way for OSA, can eliminate upper airway collapses, reduce pharynx edema, enhance functional residual capacity, improve lung compliance, reduce work of breath, airway resistanceand show its great improvement on hypoxic condition. Keeping the pressure around $8-15 \mathrm{cmH}_{2} 0$, most of the patients show great tolerance. But its efficiency and tolerability should be observed in long term use.

The flowchart of diagnosis and treatment of $\mathrm{RH}$ can be seen in Figure 1.

\section{Result and Conclusion}

Hypertension is one of the most important preventable causes for numerous diseases, especially for cardiovascular and cerebrovascular diseases. Despite the availability of effective antihypertensive drugs, BP remains resistant for too many patients. Using a systematic approach to find out the causes of $\mathrm{RH}$ and the distinctions between pseu- 
$\mathrm{RH}$ for office BP measurement

(Full-dose-full-period usage of three kinds of antihypertensive drugs, still fail to reach standard) $\downarrow$ (Measurement error?)

$\frac{\downarrow \quad(\mathrm{no})}{\downarrow} \frac{\downarrow}{\downarrow}$

Home BP Measurement or Ambulatory BP monitoring $\downarrow(\mathrm{RH} ?)$

$$
\downarrow \text { (yes) }
$$

Poor drugs adherence?

Drugs induced hypertension?

Inappropriate drugs prescription? $\downarrow$ (no)

$\downarrow(\mathrm{RH} ?)$

$$
\downarrow \text { (no) }
$$

Life intervention

Salt restriction

Alcohol restriction

Smoking cessation

Lower the body weight

Aerobic exercise

$$
\downarrow(\mathrm{RH} \text { ?) }
$$

$$
\downarrow \text { (no) }
$$

Life intervention

$\frac{\downarrow \text { (RH?) }}{\downarrow \text { (no) } \downarrow}$

Enhance drugs adherence

Discontinue or minimize interfereing substances Adjust drugs prescription

$$
\downarrow(\mathrm{RH} ?)
$$

Home BP Measurement or Ambulatory BP monitoring

\section{Pseudo RH}

(white-coat effect)

\section{Current therapies True $\mathrm{RH}$ Cushing syndrome)?}

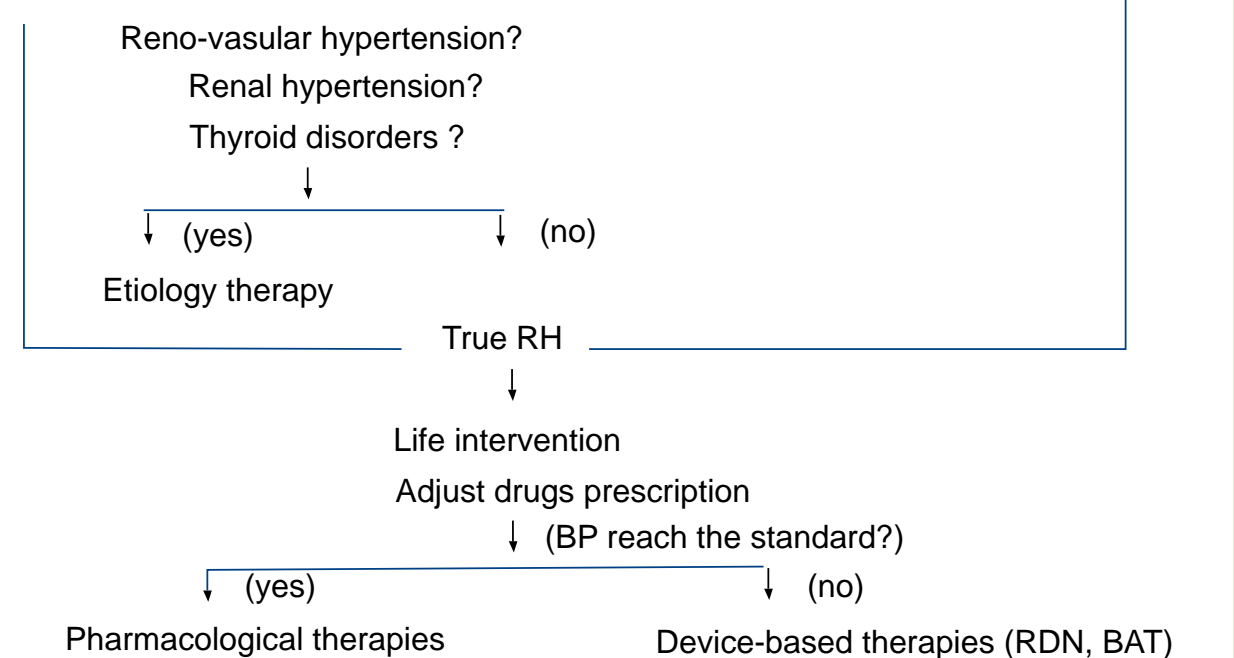

Figure 1: The diagnosis and treatment flowchart for Resistant Hypertension. 
do-resistance and true resistance are of importance for treatment. Following the current treatment guidelines and using the standardized way to establish a flowchart to manage the patients are the cor-rect methods for BP control. In the absence of novel antihypertensive drugs targeting new pathways, device-based therapies including RDN, carotid baroreflex activation and continuous positive airway pressure (CPAP) have shown encouraging results. Further efforts on the pathophysiology of $\mathrm{RH}$ will help to establish better drug combinations or new ways on BP control.

\section{References}

1. Mancia G, Fagard R, Narkiewicz K, Redon J, Zanchetti A, et al. (2013) 2013 $\mathrm{ESH} / \mathrm{ESC}$ Guidelines for the management of arterial hypertension: the Task Force for the management of arterial hypertension of the European Society of Hypertension (ESH) and of the European Society of Cardiology (ESC). J Hypertens 31: 1281-1357.

2. Persell SD (2011) Prevalence of resistant hypertension in the United States, 2003-2008. Hypertension 57: 1076-1080.

3. Barbato A, Galletti F, lacone R, Cappuccio FP, Rossi G, et al. (2012) Predictors of resistant hypertension in an unselected sample of an adult male population in Italy. Intern Emerg Med 7: 343-351.

4. Daugherty SL, Powers JD, Magid DJ, Tavel HM, Masoudi FA, et al. (2012) Incidence and prognosis of resistant hypertension in hypertensive patients. Circulation 125: 1635-1642.

5. Calhoun DA, Jones D, Textor S, Goff DC, Murphy TP, et al. (2008) Resistant hypertension: diagnosis, evaluation, and treatment. A scientific statement from the American Heart Association Professional Education Committee of the Council for High Blood Pressure Research. Hypertension 51: 1403-1419.

6. Vrijens B, Vincze G, Kristanto P, Urquhart J, Burnier M (2008) Adherence to prescribed antihypertensive drug treatments: longitudinal study of electronically compiled dosing histories. BMJ 336: 1114-1117.

7. Armario P, Oliveras A, Hernandez DRR, Ruilope LM, De La Sierra A (2011) [Prevalence of target organ damage and metabolic abnormalities in resistant hypertension]. Med Clin (Barc) 137: 435-439.

8. Pisoni R, Ahmed MI, Calhoun DA (2009) Characterization and treatment of resistant hypertension. Curr Cardiol Rep 11: 407-413.

9. Tiurenkov IN (2002) [Drug-induced hypertension and hypertensive crisis] Klin Med (Mosk) 80: 18-21.

10. Pedrosa RP, Drager LF, Gonzaga CC, Sousa MG, de Paula LK, et al. (2011) Obstructive sleep apnea: the most common secondary cause of hypertension associated with resistant hypertension. Hypertension 58: 811-817.

11. Pratt-Ubunama MN, Nishizaka MK, Boedefeld RL, Cofield SS, Harding SM (2007) Plasma aldosterone is related to severity of obstructive sleep apnea in subjects with resistant hypertension. Chest 131: 453-459.

12. Calhoun DA, Nishizaka MK, Zaman MA, Thakkar RB, Weissmann P
(2002) Hyperaldosteronism among black and white subjects with resistant hypertension. Hypertension 40: 892-896.

13. Mukherjee JJ, Khoo CM, Thai AC, Chionh SB, Pin L (2010) Type 2 diabetic patients with resistant hypertension should be screened for primary aldosteronism. Diab Vasc Dis Res 7: 6-13.

14. Nishizaka MK, Pratt-Ubunama M, Zaman MA, Cofield S, Calhoun DA (2005) Validity of plasma aldosterone-to-renin activity ratio in African American and white subjects with resistant hypertension. Am J Hypertens 18: 805-812.

15. Textor SC (2009) Current approaches to renovascular hypertension. Med Clin North Am 93: 717-732.

16. Nakano D, Mori T (2012) Salt-sensitive hypertension. Introduction. Clin Exp Pharmacol Physiol 39: 87-89.

17. Hanselin MR, Saseen JJ, Allen RR, Marrs JC, Nair KV (2011) Description of antihypertensive use in patients with resistant hypertension prescribed four or more agents. Hypertension 58: 1008-1013.

18. Hermida RC, Ayala DE, Calvo C, Lopez JE, Mojon A, et al. (2005) Effects of time of day of treatment on ambulatory blood pressure pattern of patients with resistant hypertension. Hypertension 46: 1053-1059.

19. Esler MD, Krum H, Sobotka PA, Schlaich MP, Schmieder RE (2010) Renal sympathetic denervation in patients with treatment-resistant hypertension (The Symplicity HTN-2 Trial): a randomised controlled trial. Lancet 376: 19031909.

20. Catheter-based renal sympathetic denervation for resistant hypertension: durability of blood pressure reduction out to 24 months. Hypertension 2011; 57: 911-917.

21. Steichen O, Sapoval M, Frank M, Bobrie G, Plouin PF (2012) [Renal-nerve ablation in patients with resistant hypertension: caution is still needed]. Presse Med 41: 349-357.

22. Mahfoud F, Lüscher TF, Andersson B, Baumgartner I, Cifkova R, et al. (2013) Expert consensus document from the European Society of Cardiology on catheter-based renal denervation. Eur Heart J 34: 2149-2157.

23. Medtronic announces US renal denervation pivotal trial fails to meet primary efficacy endpoint while meeting primary safety end point [press release]. January 9, 2014.

24. Hajduczok G, Chapleau MW, Johnson SL, Abboud FM (1991) Increase in sympathetic activity with age. I. Role of impairment of arterial baroreflexes. Am J Physiol 260: H1113-1120.

25. Zar T, Peixoto AJ (2008) Paroxysmal hypertension due to baroreflex failure. Kidney Int 74: 126-131.

26. Scheffers IJ, Kroon AA, Schmidli J, Jordan J, Tordoir JJ, et al. (2010) Novel baroreflex activation therapy in resistant hypertension: results of a European multi-center feasibility study. J Am Coll Cardiol 56: 1254-1258.

27. Ng MM, Sica DA, Frishman WH (2011) Rheos: an implantable carotid sinus stimulation device for the nonpharmacologic treatment of resistant hypertension. Cardiol Rev 19: 52-57. 